\title{
Mitochondrial Haplogroup Reveals the Genetic Basis of Diabetes Mellitus Type 2 Comorbidity in Psoriasis
}

\author{
Materah Salem Alwehaidah ${ }^{\mathrm{a}}$ Moiz Bakhiet ${ }^{\mathrm{b}}$ Suad AlFadhli ${ }^{\mathrm{a}}$ \\ aDepartment of Medical Laboratory, Faculty of Allied Health, Kuwait University, Sulaibekhat, Kuwait; \\ ${ }^{b}$ Department of Molecular Medicine, College of Medical and Medicine Sciences, Arabian Gulf University, Manama, \\ Kingdom of Bahrain
}

\section{Highlights of the Study}

- Patients with psoriasis (Ps) have the potential risk of comorbidity of diabetes mellitus type 2

- Haplogroup M may increase the risk of Ps

- Haplogroups R0 and J may decrease the risk of diabetes mellitus

- Diabetes comorbidity in patients with Ps is related to mitochondrial dysfunction

\section{Keywords}

Psoriasis · Diabetes mellitus type 2 - Mitochondrial

haplogroups · Mitochondria

\section{Abstract \\ Objective: Published data show a clear link between psoria- sis (Ps) and the increasing prevalence of comorbid condi- tions, such as diabetes mellitus type 2 (DM2). The role of the mitochondrial genomic haplogroup in the potential coexis- tence of Ps and DM2 comorbidity is the subject of this study. Material and Methods: Ninety-eight Kuwaiti individuals were recruited in 4 cohorts (20 healthy controls, 15 with DM2, 34 with Ps, and 29 with Ps and diabetes mellitus). An Ion Torrent S5XL was used to sequence mitochondrial DNA (mtDNA). $X^{2}$ test was used to assess differences in the distri- bution of each haplogroup between cases and controls ( $p<$ 0.05). The Bonferroni correction was applied ( $p<0.004)$. The mtDNA haplogroups were analyzed by HaploGrep. Results: Haplogroups R0, U, J, T, N, L3, M, H, X, HV, R, and K were de- tected in the studied population. Haplogroup $M$ had a high}

karger@karger.com www.karger.com/mpp

Karger $\stackrel{\text { ' }}{5}$

BOPEN ACCESS
(C) 2020 The Author(s)

Published by S. Karger AG, Basel

This is an Open Access article licensed under the Creative Commons Attribution-NonCommercial-4.0 International License (CC BY-NC) (http://www.karger.com/Services/OpenAccessLicense), applicable to the online version of the article only. Usage and distribution for commercial purposes requires written permission. risk for Ps (odds ratio (OR) 4.0, $p=0.003$ ). Haplogroup $\mathrm{R} 0$ and $\mathrm{J}$ had decreased the risk of DM2 (OR 0.28, $p=0.007)$. Conclusion: Our results indicated that mtDNA haplogroups have a potential contribution to the pathogenesis of Ps and DM2 comorbidity. We show for the first time that the comorbidity of diabetes in Ps may be related to mitochondrial dysfunction.

(c) 2020 The Author(s)

Published by S. Karger AG, Basel

\section{Introduction}

Human mitochondrial DNA (mtDNA) is a small circular double-stranded DNA molecule of $16,569 \mathrm{bp}$ in length, located in all nucleated cells. It contains 37 genes which encode 13 essential polypeptides of the oxidative phosphorylation system and the 12 and 16S rRNA and 22 tRNAs [1]. Moreover, the control region (D-loop) contains regulatory sequences controlling both replication and transcription of mtDNA that is highly susceptible to mutations because of its continuous exposure to high lev- 
els of reactive oxygen species (ROS) generated during oxidative phosphorylation. The rate of mtDNA mutation has been estimated to be 5-10 times higher than nuclear DNA due to ROS generation and lack of protective histones. Accumulative studies have reported that specific mtDNA variants have contributed to mitochondrial pathogenicity [1].

During evolution, several mutations have accumulated and scattered in the mtDNA. These mutations have subdivided the human population into several discrete, region-specific mitochondrial clades or haplogroups. Emerging evidence suggests that different mitochondrial haplogroups have a role in mitochondrial function and mitochondria-mediated signaling pathways $[2,3]$. Therefore, it has been linked to a series of metabolic diseases such as obesity, diabetes mellitus type 2 (DM2), and DM2-associated complications [4-6]. Recently, haplogroup N9a has been associated with an increased risk of DM2 and significantly associated with the incidence of diabetic nephropathy [3]. Several studies have linked mtDNA haplogroups to specific diseases such as cancer [7] and late-onset neurodegenerative disease [5].

Recently, the association between microbiome and mitochondrial function and mtDNA haplogroups has been reported. Mitochondria and microbiome both have a maternal inheritance and a circular genome. It has been shown that patients with mitochondrial diseases are more susceptible to develop bacterial infections [8]. In addition, the gut microbiota metabolites such as short-chain fatty acids may modify mitochondria activity that can activate AMP kinase and lead to mitochondrial genesis. Also, it contributes to host energy production, ROS, and inflammation modulation. Thus, mtDNA variants have a critical role in regulating the gut microbiota activity, mostly effecting intestinal barrier function and mucosal immune responses [8]. Ma et al. [9] reported that a high mutation rate of mtDNA linked with mtDNA haplogroups may influence microbiome through a potential selective and different inflammatory response to different levels of ROS activity. Also, they showed a significant association between mtDNA haplogroups and specific microbiota community [9].

Psoriasis (Ps) is a common chronic immunologically mediated inflammatory skin disease affecting approximately $2-4 \%$ of the population [10]. It plays an important role in driving insulin resistance and metabolic syndrome (MetS) [10]. The association of Ps with metabolic diseases has been studied in several populations, and previous studies have found an association between Ps and DM2 [11-13]. A meta-analysis done by Juan et al. [14] sug-

Association of Mitochondrial Haplogroup with Psoriasis and Its Comorbidity gested that Ps patients are susceptible to diabetes with odds ratio $(\mathrm{OR})=1.42$. In another study, the same association found the OR was 1.76 [11]. In a study conducted by Mala et al. [13], fasting and postprandial blood glucose, as well as glycated hemoglobin percentage (HbA1C), were significantly higher among psoriatic patients. Another study correlated the association between Ps and DM2 and atherosclerosis, reporting that the proportion of DM2 and atherosclerosis was significantly higher in the Ps group with $\mathrm{OR}=1.27$ and 1.28 , respectively. Also, the association was prominent in patients between 35 and 55 years of age [12]. However, the nature of the association between Ps and DM2 is still obscure.

Many studies have focused on the correlation between Ps and DM2 based on the examination of nuclear DNA mutation in certain susceptibility loci and investigate shared pathogenesis between these 2 diseases. In China, 89 reported diabetes susceptibility loci were genotyped in 4,456 Ps patients, and PTPN22, ST6GAL1, and JAZF1 were suggested as Ps risk genes shared pathogenesis between Ps and diabetes [15]. In another study, they evaluated the selected cardiovascular and metabolic SNPs for association with Ps. This study suggested that patients with Ps are enriched for certain common genetic variants (HLA, FUT2, UBE2L3, and SH2B3) that predispose to increased risk of MetS [16]. Polic et al. [17] evaluated the possible association between polymorphisms in the vitamin D receptor gene and the tendency for the development of Ps vulgaris and diabetes mellitus. He concluded that none of the analyzed polymorphisms individually was associated with the risk of development of Ps, diabetes, or combined phenotype [17].

Interestingly, none of the studies linked the mtDNA haplogroups with Ps comorbidity with DM2. Two studies investigated the association of European mtDNA haplogroups with patients of Ps and psoriasis arthritis (PsA). One of the studies concluded that haplogroup J was significantly less frequent among PsA patients, suggesting a protective effect, while the other one concluded that the subclades of the haplogroup U (U4a2 and U5) associated with increased risk of Ps $[18,19]$. In the current study, the genetic link between Ps and DM2 based on mtDNA haplogroups association has been examined.

\section{Material and Methods}

A total of 98 unrelated Kuwaiti subjects were recruited for this study. Patient groups consisted of 15 DM2, 34 Ps, and 29 psoriasis and diabetes mellitus type 2 (PsDM2) patients, and 20 unrelated healthy Kuwaiti subjects were included without any dermatologi- 
Table 1. Demographic parameters of cohort groups

\begin{tabular}{|c|c|c|c|c|c|c|c|c|}
\hline Gender & Ps & $p$ value $^{\mathrm{a}}$ & DM2 & $p$ value $^{\mathrm{a}}$ & PsDM2 & $p$ value $^{\mathrm{a}}$ & $\mathrm{HC}$ & $p$ value $^{\mathrm{a}}$ \\
\hline Male, \% & 58 & \multirow{4}{*}{0.24} & 46 & \multirow{4}{*}{0.25} & 58 & \multirow{4}{*}{0.05} & 50 & \multirow{4}{*}{0.07} \\
\hline Age $($ mean $\pm S D)$ & $43 \pm 18$ & & $50 \pm 8$ & & $57 \pm 9$ & & $33 \pm 10$ & \\
\hline Female, \% & 41 & & 53 & & 41 & & 50 & \\
\hline Age $($ mean $\pm S D)$ & $40 \pm 10$ & & $47 \pm 7$ & & $51 \pm 8$ & & $28 \pm 5$ & \\
\hline
\end{tabular}

SD, standard deviation; Ps, psoriasis; DM2, diabetes mellitus type 2; PsDM2, psoriasis diabetes mellitus type 2; HC, healthy control. ${ }^{a} t$ test $(p<0.05)$.

cal or metabolic disease. Written consent was obtained from all participants, following a protocol approved by Kuwait University and the Ministry of Health.

Genomic DNA was extracted from whole blood using a QIAamp DNA Blood Mini Kit (Qiagen, Venlo, The Netherlands) according to the manufacturer's instructions. Each DNA sample was checked for purity using a NanoDrop 1000 system (Thermo Fisher Scientific) and for concentration using a Qubit 3.0 Fluorometer (Thermo Fisher Scientific). The concentration of input DNA was then adjusted to $1 \mathrm{ng} / \mu \mathrm{L}$. mtDNA whole genome sequence was done by Ion Torrent S5XL according to precision ID mtDNA panels with the HID Ion S5 ${ }^{\mathrm{TM}} / \mathrm{HID}$ Ion Gene Studio ${ }^{\mathrm{TM}} \mathrm{S} 5$ System application guide. Raw signal data from sequencing runs from the Ion Torrent S5XL were automatically transferred to the Torrent Server Hosting the Torrent Suite Software that processed the raw voltage semiconductor sequencing data into DNA base calls. The pipeline included signaling processing, base calling, quality score assignment, adapter trimming, read mapping to 19 reference human genomes, quality control of mapping quality, coverage analysis with down sampling, and variant calling. The identification of variants was performed by the Ion Torrent Variant Caller plug-in and Ion Reporter Software v5.2 (Life Technologies). Torrent Variant Caller v5.2 was used for alignment and variant detection according to the revised Cambridge Reference Sequence of humans [20]. Samples were multiplexed and sequenced on an Ion 520 chip (3-6 megabase throughput). The average throughput of Ion 520 chip was $3.5 \mathrm{Mb}$. The average coverage depth was $24,625.2 \times$ and the mean read length was $144 \mathrm{bp}$. However, the average total reads were 3,359,441.

SPSS 15.0 software (SPSS Inc., Chicago, IL, USA) package was used for the statistical analysis. The demographic comparison of groups was performed using the $t$ test. Pearson's $\chi^{2}$ test was used to assess differences in the distribution of each haplogroup between cases and controls. The results were evaluated with $95 \%$ CLs and $p$ value $<0.05$ was considered as significant. Because of the multiple comparisons of mtDNA haplogroups, the Bonferroni correction was applied. We examined 12 haplogroups (R0, U, J, T, N, L3, M, H, X, HV, R, and K) and divided 0.05 by 12 to give 0.004 . Thus, a $p$ value of $<0.004$ was considered statistically significant. We used the HaploGrep program to annotate the mtDNA haplogroup for the cases and controls; however, the haplogroup quality scores reported by HaploGrep was $95 \%[21]$.
Table 2. Distribution of mtDNA haplogroups among the studied cohort groups

\begin{tabular}{lllll}
\hline Haplogroups & \multicolumn{4}{l}{ Frequency, $\%$} \\
\cline { 2 - 5 } & $\begin{array}{l}\mathrm{HC} \\
(n=20)\end{array}$ & $\begin{array}{l}\text { Ps } \\
(n=34)\end{array}$ & $\begin{array}{c}\text { DM2 } \\
(n=15)\end{array}$ & $\begin{array}{l}\text { PsDM2 } \\
(n=29)\end{array}$ \\
\hline R0 & 20 & 9 & 7 & 10 \\
U & 20 & 9 & 27 & 24 \\
J & 20 & 15 & 7 & 14 \\
T & 10 & 6 & 20 & 3 \\
N & 5 & 3 & 0 & 0 \\
L3 & 10 & 18 & 20 & 14 \\
M & 5 & 18 & 0 & 10 \\
H & 0 & 12 & 13 & 7 \\
X & 0 & 0 & 7 & 3 \\
HV & 0 & 3 & 0 & 3 \\
R & 0 & 6 & 0 & 7 \\
K & 10 & 3 & 0 & 3 \\
\hline
\end{tabular}

Ps, psoriasis; DM2, diabetes mellitus type 2; PsDM2, psoriasis diabetes mellitus type 2; HC, healthy control; mtDNA, mitochondrial DNA.

\section{Results}

\section{MtDNA Haplogroup Distribution}

Using Ion Torrent S5XL next-generation sequencing, mtDNA haplogroups were analyzed in 98 individuals from the Kuwaiti population subdivided into 4 cohorts; 15 DM2, or 34 Ps, 29 PsDM2, and 20 healthy controls (HCs). Table 1 shows the demographic parameters of the cohort groups. There were no significant differences between the age of females and males among the study groups.

Twelve haplogroups were detected in the 4 cohorts (R0, U, J, T, N, L3, M, H, X, HV, R, and K). The frequencies of the haplogroups found in the various cohorts are summarized in Table 2. The absence of mitochondrial 
Table 3. Multivariate statistical analysis for the various haplogroups in the disease cohorts versus healthy controls

\begin{tabular}{|c|c|c|}
\hline Haplogroups & $p$ value $^{\mathrm{a}}$ & OR (RR) $(95 \% \mathrm{CI})$ \\
\hline \multicolumn{3}{|l|}{ a. Ps group } \\
\hline R0 & 0.02 & $0.36(0.4)(0.1-0.9)$ \\
\hline $\mathrm{U}$ & 0.02 & $0.36(0.4)(0.1-0.9)$ \\
\hline $\mathrm{J}$ & 0.46 & \\
\hline $\mathrm{T}$ & 0.3 & \\
\hline L3 & 0.15 & \\
\hline M & 0.003 & $4(3)(1.2-2.8)$ \\
\hline K & 0.04 & $0.3(0.3)(0.07-1)$ \\
\hline \multicolumn{3}{|l|}{ b. DM2 group } \\
\hline R0 & 0.007 & $0.2(0.3)(0.1-0.7)$ \\
\hline $\mathrm{U}$ & 0.32 & \\
\hline $\mathrm{J}$ & 0.007 & $0.2(0.3)(0.1-0.7)$ \\
\hline $\mathrm{T}$ & 0.04 & $2(2)(0.6-2.7)$ \\
\hline L3 & 0.05 & \\
\hline \multicolumn{3}{|l|}{ c. PsDM2 } \\
\hline R0 & 0.04 & $0.4(0.8)(0.1-1)$ \\
\hline $\mathrm{U}$ & 0.61 & \\
\hline $\mathrm{J}$ & 0.34 & \\
\hline $\mathrm{T}$ & 0.04 & $0.2(0.3)(0.07-1)$ \\
\hline L3 & 0.51 & \\
\hline M & 0.28 & \\
\hline K & 0.04 & $0.2(0.3)(0.07-1)$ \\
\hline \multicolumn{3}{|c|}{ d. Ps and PsDM2 groups } \\
\hline R0 & 0.04 & $0.4(0.8)(0.1-1)$ \\
\hline $\mathrm{U}$ & 0.4 & \\
\hline $\mathrm{J}$ & 0.2 & \\
\hline $\mathrm{T}$ & 0.2 & \\
\hline $\mathrm{N}$ & 0.09 & \\
\hline L3 & 0.2 & \\
\hline $\mathrm{M}$ & 0.02 & $3(1.1)(1-1.2)$ \\
\hline K & 0.04 & $0.4(0.8)(0.1-1)$ \\
\hline
\end{tabular}

OR, odds ratio; RR, risk ratio; Ps, psoriasis; DM2, diabetes mellitus type 2; PsDM2, psoriasis diabetes mellitus type $2 .{ }^{a} \chi^{2}$ test $(p$ value $<0.05)$.

haplogroup $\mathrm{H}$ in HCs compared to Ps (12\%), PsDM2 (7\%), and DM2 (13\%) suggests that haplogroup H is a risk factor of Ps and DM2. The presence of haplogroup X in groups PsDM2 and DM2 (3 and 7\%, respectively) and absence in $\mathrm{HC}$ and Ps groups suggested that the haplogroup X may be associated with DM2 disease. Conversely, the absence of haplogroups $\mathrm{HV}$ and $\mathrm{R}$ in $\mathrm{HC}$ and DM2 groups and its presence in PsDM2 and Ps groups (7 and $6 \%$, respectively, and $\mathrm{HV}=3 \%$ in both) suggested that haplogroups $\mathrm{HV}$ and $\mathrm{R}$ may associate with Ps (Table 2). The small sample size of haplogroup $\mathrm{X}, \mathrm{HV}$, and $\mathrm{R}$ in the study groups may affect the significance of statisti- cal analysis. Therefore, it needs to be investigated with a larger sample size.

While comparing the frequency of haplogroups found in Ps patients against healthy control, our results show that the frequency of haplogroups $\mathrm{R} 0$ and $\mathrm{U}$ had the same effect ( 9 vs. $20 \%, \mathrm{OR}=0.36,95 \% \mathrm{CI}=0.1-0.9$, with $p=$ 0.02 and risk ratio $[R R]=0.4)$ and was significantly higher in healthy controls than Ps patients. Following the same trend, haplogroup K was significantly higher in $\mathrm{HCs}$ than in Ps $(10$ vs. $3 \%, \mathrm{OR}=0.3,95 \% \mathrm{CI}=0.07-1$, with $p=0.04$ and $\mathrm{RR}=0.3)$. Haplogroup $\mathrm{M}$ had an opposite effect, where the frequency was ( 18 vs. $5 \%, \mathrm{OR}=4.0,95 \%$ $\mathrm{CI}=1.2-2.8$, with $p=0.003$ and $\mathrm{RR}=3$ ) significantly lower in the healthy controls than the Ps group (Table $3 a$ ).

When comparing the frequency of haplogroups found in DM2 patients against healthy controls, our results also show that the frequency of haplogroups R0 and J but not $\mathrm{U}$ (7 vs. $20 \%$, OR $=0.2,95 \% \mathrm{CI}=0.1-0.7$, with $p=0.007$ and $R R=0.3$ ) was significantly higher in the healthy controls than DM2 patients. In contrast, haplogroup T had an opposite effect where the frequency of haplogroups $\mathrm{T}$ (20 vs. $10 \%, \mathrm{OR}=2,95 \% \mathrm{CI}=0.6-2.7$, with $p=0.04$ and $\mathrm{RR}=2$ ) was significantly lower in healthy controls than DM2 (Table 3b). For further analysis, the cluster of haplogroup JT of DM2 was analyzed. The frequency of haplogroup JT was 26 and 30\% in DM2 and healthy controls, respectively, with $p=0.5$. When comparing the frequency of the haplogroups found in PsDM2 against the healthy controls, our results also show that the frequency of haplogroups R0 ( 10 vs. $20 \%$, OR $=0.4,95 \% \mathrm{CI}=0.1-1$, with $p=0.04$ and $\mathrm{RR}=0.8)$ and both haplogroups $\mathrm{T}$ and $\mathrm{K}$ ( 3 vs. $10 \%, \mathrm{OR}=0.2,95 \% \mathrm{CI}=0.07-1$, with $p=0.04$ and $\mathrm{RR}=0.3$ ) was significantly higher in the healthy controls than in the PsDM2 patients (Table 3c).

In this study, the sample size of the study groups was small that may affect the statistical power. To investigate a large sample size, the mtDNA haplogroups of Ps and PsDM2 groups were combined and compared with healthy control (Table 3d). The frequency of the haplogroups $\mathrm{R} 0$ and $\mathrm{K}$ found in combination of $\mathrm{Ps}$ and PsDM2 groups was higher in healthy controls (20 vs. $10 \%$, $\mathrm{OR}=0.4,95 \% \mathrm{CI}=0.1-1$, with $p=0.04$ and $\mathrm{RR}=0.8$ ), while haplogroup $\mathrm{M}$ was higher in the combined group of Ps and PsDM2 (14 vs. 5\%, OR =3, 95\% CI $=1-1.2$, with $p=0.02$ and $\mathrm{RR}=1.1)$.

Furthermore, the haplogroups were clustered into macro-haplogroup (L3, M, and N) according to the phylogenetic tree [22]. The statistical analysis of haplogroup L3 and M were mentioned previously. Macro-haplogroup $\mathrm{N}$ was significantly higher in healthy controls than in Ps 
and PsDM2 groups as well as the combination of these groups ( $p=0.0006 ; 0.07$ and 0.007 , respectively). These results, after the Bonferroni correction (setting at $p<$ 0.004 ), showed a significant relationship between the generation of Ps and subjects carrying mitochondrial haplogroup M (Table 3a), while a protective effect of macrohaplogroup $\mathrm{N}$ against Ps and also a borderline protective effect of haplogroup R0 and J against DM2 (Table 3b).

\section{Discussion}

In the present study, frequencies of mtDNA haplogroups were determined in patients with Ps and Ps patients who develop DM2 comorbidity during the interval of the disease. In addition, this study reveals the mtDNA genetic basis of DM2 comorbidity in psoriatic patients. Our data show an association of mtDNA haplogroups with Ps, diabetes mellitus, and combined Ps with DM2.

This study suggests that haplogroup $\mathrm{M}$ was significantly more frequent in Ps than in controls and may increase the risk of Ps. Haplogroup M is defined by 263, 489, $10,400,14,783$, and 15,043 mutations motif (www.snpedia.com/index.php/MtDNA_haplogroup). There is a G15043A variant located in the MT-CYB gene. This gene encodes the protein cytochrome $b$ which plays a role in the function of the complex III of the mitochondrial respiratory chain. The G15043A variant correlated with metabolic and psychiatric diseases in a different study [23]. Because of the G15043A variant associated with the metabolic disease [24], its effect may be associated with Ps. According to the literature, we know of only 2 studies that investigated the association of common European mtDNA haplogroups with patients of Ps and PsA. One of which concluded that haplogroup J was significantly less frequent among PsA patients, suggesting a protective effect, while the other 1 suggested that the U4a2 and U5 associated with increased risk of Ps $[18,19]$.

Another possible susceptible haplogroup is T, which was significantly more frequent in DM2. Haplogroup T is defined by $709,1,888,4,917,8,697,10,463,13,368,14,905$, $15,607,15,928$, and 16,294 mutations motif (www.snpedia. com/index.php/MtDNA_haplogroup). Thereis an A4917G variant located in the MT-ND2 gene. MT-ND2 (NADH dehydrogenase 2) is a core subunit of the mitochondrial NADH dehydrogenase (complex I) that catalyzes the transfer of electrons from $\mathrm{NADH}$ to ubiquinone by pumping protons through the mitochondrial membrane complexes to provide energy for the generation of ATP.
Mutations within the NADH dehydrogenase 2 mitochondrial gene have been found in patients with the mitochondrial type of Alzheimer disease, Leber hereditary optic neuropathy and DM2 [25,26]. Crispim et al. [27] studied the mtDNA m.4216T $>$ C and m.4917A $>$ G variants, which are European-specific mitochondrial cluster $\mathrm{J} / \mathrm{T}$ in the development of type 2 diabetes mellitus in Caucasian-Brazilian patients. He found that the frequency of these variants is higher in diabetic patients than in control subjects. Moreover, haplogroups J, defined by the presence of the m.4216T $>\mathrm{C}$ variant only, and $\mathrm{T}$, defined by the presence of both m.4216T $>\mathrm{C}$ and $\mathrm{m} .4917 \mathrm{~A}>\mathrm{G}$ variants, are more frequent in the diabetic group than in the control group [27]. In this study, the haplogroup JT was not significantly associated with DM2, while haplogroup T was significantly linked to DM2 but not haplogroup J.

In Taiwan, haplogroup B was associated with an increased risk of diabetes generation, whereas those harboring mitochondrial haplogroup $\mathrm{D}$ were found to have a resistance to the development of DM2 [4]. However, mtDNA haplogroup N9a was associated with an increased occurrence of MD2 and significantly associated with diabetic nephropathy incidence [3]. Also, Kofler et al. [28] has reported that $\mathrm{mtDNA}$ haplogroup $\mathrm{T}$ is associated with coronary artery disease and diabetic retinopathy.

The frequency of haplogroup X for DM2 and PsDM2 groups was 7 and 3\%, respectively, while the frequency of $\mathrm{HC}$ and Ps groups was $0 \%$, suggesting that haplogroup X may be a risk factor of DM2 and for developing diabetes in Ps patients for the duration of the disease. This result may need to confirm with a large sample size as the low frequency of haplogroup $\mathrm{X}$ in study groups may obscure the rational distribution.

Haplogroup $\mathrm{X}$ is defined by $73,7,028,11,719,12,705$, $14,766,16,189,16,223$, and 16,278 mutations motif (www. snpedia.com/index.php/MtDNA_haplogroup). There is a $\mathrm{T}>\mathrm{C}$ transition in the 16,189 -bp position of the noncoding control region of mtDNA that has been associated in several studies with DM2, MetS, and obesity [6]. This variant resides in the first hypervariable segment of the control region of mtDNA, containing a homopolymeric tract of cytosines, which is interrupted by a thymidine at nucleotide position 16,189 . T to $C$ substitution at position 16,189 creates an uninterrupted tract of 8-12 cytosines (poly-C tract) [29]. The T16189C variant has been associated with thinness at birth and impaired glucose tolerance [30]. Poulton et al. [31] reported a significant association of T16189C with higher fasting insulin levels and insulin resistance. Likewise, Liou et al. [32] concluded that the 
T16189 C variant can impact the development of DM2 in combination with high BMI. A similar association with MetS has been confirmed by another study of Chinese population [33].

There are at least 25 mitochondrial variants that have been associated with DM2. Most of these variants are rare and are associated with additional traits such as neurological symptoms or myopathy. One common variant, T16189C, has been reported to be associated with DM2, a higher fasting insulin level and lower body mass in infants [34]. Interestingly, in this study, haplogroup T was susceptible to DM2, while there was also a protective effect with PsDM2. This suggests that the DM2 comorbidity in Ps patients may be related to mitochondrial dysfunction.

This is the first study to correlate mtDNA haplogroups with Ps comorbidity with DM2 and investigate the $\mathrm{mtD}$ NA haplogroups of Ps patients in Kuwaiti population. The limitation of this study was the small sample size of study groups that may weaken the statistical analysis.

\section{Conclusion}

This study suggests that mtDNA haplogroups have a potential contribution to the pathogenesis of Ps as a disease and DM2 as a comorbidity of Ps which is related to mitochondrial dysfunction. This study showed the potential risk of haplogroup $\mathrm{M}$ and the protective effect of macro-haplogroup $\mathrm{N}$ in Ps.

\section{Acknowledgements}

The authors thank Bader Sultan and Brothers Co., Kuwait, for offering substantial discounts on reagent kits and Ms. Rita C. for editing the article.

\section{Statement of Ethics}

A written permission was obtained from each participant under the protocols approved by the joint Committee for the Protection of Human Subjects in Research in Kuwait (KIMS). This research was confirmed by the Health Science Center Ethics Committee at Kuwait University and Health and Medical Research Committee in the Ministry of Health and registered on No. 2016/496.

\section{Conflict of Interest Statement}

The authors declare no conflicts of interest.

\section{Funding Sources}

This work was supported by Kuwait University.

\section{Author Contributions}

Conceptualization: Materah Salem Alwehaidah and Suad AlFadhli; data curation, formal analysis, investigation, methodology, project administration, resources, software, validation, visualization, project administration, and writing original draft: Materah Salem Alwehaidah; and supervision: Materah Salem Alwehaidah, and Moiz Bakhiet.

\section{References}

1 Tanaka M, Takeyasu T, Fuku N, Li-Jun G, Kurata M. Mitochondrial genome single nucleotide polymorphisms and their phenotypes in the Japanese. Ann N Y Acad Sci. 2004;1011:7-20.

2 Kuo HM, Weng SW, Chang AY, Huang HT, Lin HY, Chuang JH, et al. Altered mitochondrial dynamics and response to insulin in cybrid cells harboring a diabetes-susceptible mitochondrial DNA haplogroup. Free Radic Biol Med. 2016;96:116-29.

3 Fang $\mathrm{H}$, Hu N, Zhao Q, Wang B, Zhou $\mathrm{H}$, Fu Q, et al. mtDNA Haplogroup N9a increases the risk of type 2 diabetes by altering mitochondrial function and intracellular mitochondrial signals. Diabetes. 2018;67(7):1441-53.

4 Liou CW, Chen JB, Tiao MM, Weng SW, Huang TL, Chuang JH, et al. Mitochondrial DNA coding and control region variants as genetic risk factors for type 2 diabetes. Diabetes. 2012;61(10):2642-51.

5 Takasaki S. Mitochondrial haplogroups associated with Japanese centenarians, Alzhei- mer's patients, Parkinson's patients, type 2 diabetic patients and healthy non-obese young males. J Genet Genomics. 2009;36(7): 425-34.

6 Aral C, Akkiprik M, Caglayan S, Atabey Z, Ozişik G, Bekiroglu N, et al. Investigation of relationship of the mitochondrial DNA 16189 $\mathrm{T}>\mathrm{C}$ polymorphism with metabolic syndrome and its associated clinical parameters in Turkish patients. Hormones. 2011;10(4): 298-303.

7 Darvishi K, Sharma S, Bhat AK, Rai E, Bamezai RN. Mitochondrial DNA G10398A polymorphism imparts maternal haplogroup $\mathrm{N}$ a risk for breast and esophageal cancer. Cancer Lett. 2007;249(2):249-55.

8 Clark A, Mach N. The crosstalk between the gut microbiota and mitochondria during exercise. Front Physiol. 2017;8:319.

9 Ma J, Coarfa C, Qin X, Bonnen PE, Milosavljevic A, Versalovic J, et al. mtDNA haplogroup and single nucleotide polymor- phisms structure human microbiome communities. BMC Genomics. 2014;15:257.

10 Oliveira MdeF, Rocha BdeO, Duarte GV. Psoriasis: classical and emerging comorbidities. An Bras Dermatol. 2015;90(1):9-20.

11 Coto-Segura P, Eiris-Salvado N, GonzálezLara L, Queiro-Silva R, Martinez-Camblor P, Maldonado-Seral C, et al. Psoriasis, psoriatic arthritis and type 2 diabetes mellitus: a systematic review and meta-analysis. Br J Dermatol. 2013;169(4):783-93.

12 Shapiro J, Cohen AD, David M, Hodak E, Chodik G, Viner A, et al. The association between psoriasis, diabetes mellitus, and atherosclerosis in Israel: a case-control study. J Am Acad Dermatol. 2007;56(4): 629-34.

13 Mala P, Bhattacharjee I, Bhattacharya G, Ghosh S, Sarker G, Pal R. Association between psoriasis, diabetes mellitus, hypertension and obesity. Clin Epidemiol Glob Health. 2015;3: 132-6. 
14 Cheng J, Kuai D, Zhang L, Yang X, Qiu B. Psoriasis increased the risk of diabetes: a metaanalysis. Arch Dermatol Res. 2012;304(2): 119-125.

15 Wang H, Wang Z, Rani PL, Fu X, Yu W, Bao F, et al. Identification of PTPN22, ST6GAL1 and JAZF1 as psoriasis risk genes demonstrates shared pathogenesis between psoriasis and diabetes. Exp Dermatol. 2017;26(11): 1112-7.

16 Lu Y, Chen H, Nikamo P, Qi Low H, Helms C, Seielstad M, et al. Association of cardiovascular and metabolic disease genes with psoriasis. J Invest Dermatol. 2013;133(3):836-9.

17 Polic MV, Rucevic I, Barisic-Drusko V, Miskulin M, Glavas-Obrovac L, Stefanic M, et al. Polymorphisms of vitamin D receptor gene in the population of eastern Croatia with psoriasis vulgaris and diabetes mellitus. Coll Antropol. 2012;36(2):451-7.

18 Coto-Segura P, Santos-Juanes J, Gómez J, Alvarez V, Díaz M, Alonso B, et al. Common European mitochondrial haplogroups in the risk for psoriasis and psoriatic arthritis. Genet Test Mol Biomarkers. 2012;16(6):621-3.

19 Hudson G, Gomez-Duran A, Wilson IJ, Chinnery PF. Recent mitochondrial DNA mutations increase the risk of developing common late-onset human diseases. PLoS Genet. 2014;10(5):e1004369.

20 Bandelt HJ, Kloss-Brandstätter A, Richards MB, Yao YG, Logan I. The case for the continuing use of the revised Cambridge reference sequence (rCRS) and the standardization of notation in human mitochondrial DNA studies. J Hum Genet. 2014;59(2):6677.
21 Weissensteiner H, Pacher D, Kloss-Brandstätter A, Forer L, Specht G, Bandelt HJ, et al. HaploGrep 2: mitochondrial haplogroup classification in the era of high-throughput sequencing. Nucleic Acids Res. 2016;44(W1): W58-63.

22 van Oven M. PhyloTree build 17: growing the human mitochondrial DNA tree. Forensic Sci Int Genet Suppl Ser. 2015;5:e392-4.

23 Rollins B, Martin MV, Sequeira PA, Moon EA, Morgan LZ, Watson SJ, et al. Mitochondrial variants in schizophrenia, bipolar disorder, and major depressive disorder. PLoS One. 2009;4(3):e4913.

24 McInnes J. Mitochondrial-associated metabolic disorders: foundations, pathologies and recent progress. Nutr Metab. 2013;10(1):63.

25 Canter JA, Olson LM, Spencer K, SchnetzBoutaud N, Anderson B, Hauser MA, et al. Mitochondrial DNA polymorphism A4917G is independently associated with age-related macular degeneration. PLoS One. 2008;3(5): e2091.

26 Savontaus ML. mtDNA mutations in Leber's hereditary optic neuropathy. Biochim Biophys Acta. 1995;1271(1):261-3.

27 Crispim D, Canani LH, Gross JL, Tschiedel B, Souto KE, Roisenberg I. The European-specific mitochondrial cluster $\mathrm{J} / \mathrm{T}$ could confer an increased risk of insulin-resistance and type 2 diabetes: an analysis of the m.4216T > $\mathrm{C}$ and m.4917A > G variants. Ann Hum Genet. 2006;70(Pt 4):488-95.

28 Kofler B, Mueller EE, Eder W, Stanger O, Maier R, Weger M, et al. Mitochondrial DNA haplogroup $\mathrm{T}$ is associated with coronary artery disease and diabetic retinopathy: a case control study. BMC Med Genet. 2009;10:35.
29 Chinnery PF, Elliott HR, Patel S, Lambert C, Keers SM, Durham SE, et al. Role of the mitochondrial DNA 16184-16193 poly-C tract in type 2 diabetes. Lancet. 2005;366(9497): 1650-1.

30 Poulton J, Marchington DR, Scott-Brown M, Phillips DI, Hagelberg E. Does a common mitochondrial DNA polymorphism underlie susceptibility to diabetes and the thrifty genotype? Trends Genet. 1998;14(10):387-9.

31 Poulton J, Bednarz AL, Scott-Brown M, Thompson C, Macaulay VA, Simmons D. The presence of a common mitochondrial DNA variant is associated with fasting insulin levels in Europeans in Auckland. Diabet Med. 2002;19(11):969-71.

32 Liou CW, Lin TK, Huei Weng H, Lee CF, Chen TL, Wei YH, et al. A common mitochondrial DNA variant and increased body mass index as associated factors for development of type 2 diabetes: additive effects of genetic and environmental factors. J Clin Endocrinol Metab. 2007;92(1):235-9.

33 Liao WQ, Pang Y, Yu CA, Wen JY, Zhang YG, Li XH. Novel mutations of mitochondrial DNA associated with type 2 diabetes in Chinese Han population. Tohoku J Exp Med. 2008;215(4):377-84.

34 Casteels K, Ong K, Phillips D, Bendall H, Pembrey M. Mitochondrial 16189 variant, thinness at birth, and type-2 diabetes. ALSPAC study team. Avon longitudinal study of pregnancy and childhood. Lancet. 1999; 353(9163):1499-500 